\begin{tabular}{|r|l|}
\hline \multicolumn{2}{|c|}{ Statistica Sinica Preprint No: SS-2021-0167 } \\
\hline Title & $\begin{array}{l}\text { Defining And Estimating Principal Stratum Specific } \\
\text { Natural Mediation Effects With Semi-competing Risks } \\
\text { Data }\end{array}$ \\
\hline Manuscript ID & SS-2021-0167 \\
\hline URL & http://www.stat.sinica.edu.tw/statistica/ \\
\hline DOI & $10.5705 /$ ss.202021.0167 \\
\hline Complete List of Authors & $\begin{array}{l}\text { Fei Gao, } \\
\text { Fan Xia and } \\
\text { Kwun Chuen Gary Chan }\end{array}$ \\
\hline Corresponding Author & Fei Gao \\
\hline E-mail & fgao@fredhutch.org \\
\hline Notice: Accepted version subject to English editing.
\end{tabular}


Statistica Sinica

\title{
DEFINING AND ESTIMATING PRINCIPAL STRATUM SPECIFIC NATURAL MEDIATION EFFECTS WITH SEMI-COMPETING RISKS DATA
}

\author{
Fei Gao ${ }^{1}$, Fan Xia ${ }^{2}$ and K. C. G. Chan ${ }^{2}$ \\ ${ }^{1}$ Fred Hutchinson Cancer Research Center and ${ }^{2}$ University of Washington
}

Abstract: In many medical studies, an ultimate failure event such as death is likely

to be affected by the occurrence and timing of other intermediate clinical events.

Both event times are subject to censoring by loss-to-follow-up but the nonterminal event may further be censored by the occurrence of the primary outcome, but not vice versa. To study the effect of an intervention on both events, the intermediate event may be viewed as a mediator, but conventional definition of direct and indirect effects is not applicable due to semi-competing risks data structure. We define three principal strata based on whether the potential intermediate event occurs before the potential failure event, which allow proper definition of direct and indirect effects in one stratum whereas total effects are defined for all strata. We discuss the identification conditions for stratum-specific effects, and propose a semiparametric estimator based on a multivariate logistic stratum membership model and within-stratum proportional hazards models for the event times. By treating the unobserved stratum membership as a latent variable, we propose an EM algorithm for computation. We study the asymptotic properties of the 
estimators by the modern empirical process theory and examine the performance of the estimators in numerical studies.

Key words and phrases: Illness-death model, Missing data, Principal stratification, Proportional hazards model, Survival analysis.

\section{Introduction}

Evaluating the causal effects of an intervention on a clinical outcome is a common theme in many medical studies. After an overall relationship between the intervention and outcome is established, it is often of further interest to understand the biological or mechanistic pathways that contribute to the causal treatment effect. Causal mediation analysis is often utilized to disentangle the total treatment effect by decomposing it into the indirect effect, i.e., the effect exerted by intermediate variables (mediators), and the direct effect, i.e., the effect involving pathways independent of the hypothesized mediators. Intuitively, to evaluate a direct effect, the mediators need to be somehow fixed. A variety of mediation effects can be defined through different ways of fixing the mediators. The natural effects are most relevant in studying treatment effect mechanisms in public health, because it compares the outcome under which mediators are set to the value that naturally occurs under different treatment assignment. A number of meth- 
ods were proposed for causal mediation analysis with survival outcomes, for a single mediator measured at study entry (Lange and Hansen, 2011; VanderWeele, 2011; Tchetgen Tchetgen, 2011; Lange et al., 2012) and for longitudinal mediators (Lin et al., 2017; Zheng and van der Laan, 2017; Didelez, 2019; Vansteelandt et al., 2019; Aalen et al., 2020).

In many biomedical studies, intermediate, non-terminal landmark events are recorded in addition to the primary failure event because they are important to evaluate prognosis. Due to the ordering of the two events, the non-terminal event is subject to censoring by the occurrence of the terminal event, but not vice versa, such that semi-competing risks data are observed (Fine et al., 2001). In this paper, we consider a setting where a non-terminal event may serve as a mediator for individuals to whom the event would occur before the terminal event. An example is a multi-center trial of allogeneic bone marrow transplants in patients with acute leukemia (Copelan et al., 1991; Klein and Moeschberger, 2006), where the primary interest is on the effect of different treatment regimen (methotrexate + cyclosporine vs methylprednisolone + cyclosporine) on the survival time. The event time of an intermediate endpoint, chronic graft-versus-host disease (GVHD), is a major side effect of the transplant that can be lethal. However, some patients died without experiencing GVHD, such that GVHD 
event time is subject to censoring by the death time.

Causal mediation analysis with semi-competing risks data is particularly challenging. First, the mediator is only well-defined for those who would have the non-terminal event developed before the occurrence of the primary event. Therefore, the conventional definition of natural indirect and direct effects based on replacing the counterfactual of mediator under one treatment by that under the other can hardly apply to the entire population. Moreover, the semi-competing risks data structure, that is, the primary event may censor the intermediate event but not vice versa, posts additional challenges in the identifiability of parameters relating to natural direct effect.

There is a surge in recent literature that tries to address various causal inference problems with semi-competing risk data. However, most of them are different from our work because they only consider subgroup average treatment effects Comment et al., 2019; Xu et al., 2020; Nevo and Gorfine, 2020) and not mediation effects. Upon finishing this paper, we became aware of the newly accepted paper by Huang (2020) which considered estimation of natural mediation effects for semi-competing risk data by a counting process framework. The problem formulation, estimand and assumptions are all very different from our work. For instance, we do not 
make sequential ignorability assumptions on surviving subpopulations at arbitrary post-treatment time as assumed in Huang (2020), because those evolving subpopulations are generally healthier than the baseline study population before the treatment is assigned.

In this paper, we consider a novel principal stratification approach to define causal mediation effects in the subgroup where the intermediate event happens when given either treatment, i.e., those susceptible to the intermediate event under both treatments. The notations and settings are given in Sections 2.1 and 2.2. We discuss the identification conditions needed for estimating the stratum-specific natural indirect and direct effects in Section 2.3. and propose a semiparametric estimator based on a multivariate logistic stratum membership model and within-stratum proportional hazards models for the event times in Section 2.4. By treating the unobserved stratum membership as a latent variable, we propose an EM algorithm for computation of the nonparametric maximum likelihood estimator in Section 2.5 . We also study the asymptotic properties of the estimators using the modern empirical process theory in Section 2.6 and examine the performance of the estimators in simulation studies Section 3. An analysis of data from a clinical trial is given in Section 4, and concluding remarks are included in Section 5. Proofs, detailed derivations, and additional numerical results 
are given in the online supplement. The computation codes for simulation studies are available at https://github.com/feigao1/Med_Semi_Comp.

\section{Methods}

\subsection{Notations for observed data}

Let $A$ be a binary treatment, $T$ be the time to a primary event of interest and $M$ be the time to an intermediate, non-terminal event. The intermediate event time $M$ may be censored by the occurrence of the primary event, but not vice versa, such that we observe semi-competing risks data. For example, $A$ is a treatment for prolonging survival time, $T$ is the time to death, and $M$ is the time to cancer progression. The occurrence of death may censor the cancer progression onset, but not vice versa.

Let $\boldsymbol{X}$ be a collection of baseline covariates that may be associated with either or both events. Let $C$ denote a censoring time for the primary event, for example, end of follow-up time. Then, we observe $Y \equiv \min (T, C)$ and $\Delta^{T}=I(T \leq C)$ for the primary event, and $Z \equiv \min (M, Y)$ and $\Delta^{M}=I(M \leq Y)$ for the intermediate event. The observations are versions of the counterfactual variables that we define as follows. 
2.2 Counterfactuals and causal estimands

\subsection{Counterfactuals and causal estimands}

To define causal mediation effects of interest, we adopt the potential outcomes framework. Let the variables $M(a)$ and $T(a) \equiv T(a, M(a))$ denote the counterfactual nonterminal and terminal event time when the treatment is set to $a$. These quantities are called single-world variables since the intervention is set to a single realizable value, in contrast with crossworld variables to be defined later which involves interventions to be set to different values. In conventional settings, $M(a)$ and $T(a)$ can be defined separately as the values of $M$ and $T$ that would be observed had the treatment been set to $a$. For semi-competing risk settings, however, the value for $M(a)$ cannot vary arbitrarily: when the potential primary event happens before the potential intermediate event, the value of the mediator is not well-defined (and is often set to $\infty$ by convention) and in such a case the potential primary event time shall not be dependent on an arbitrary $m$ greater than the potential primary event time. Therefore, to be consistent with the semi-competing risk data structure, we have the following order invariance that either $M(a)<\infty$ and $T(a, M(a)) \geq M(a)$, or $M(a)=\infty$ and thereby $T(a, M(a))<M(a)$.

In conventional mediation analysis, a comparison between the potential outcome with the mediator set to different values would define an indirect 
2.2 Counterfactuals and causal estimands

effect. Furthermore, if the mediators are set to counterfactual values under different interventions, the indirect effect is called the natural indirect effect. On the other hand, if the mediators are both set to the same value, a comparison between the potential outcomes with different interventions would define a direct effect. Like the natural indirect effect, if the mediators are set to the counterfactual value under the same intervention, the direct effect is called the natural direct effect. Both natural indirect and direct effects involve the term $T\left(a^{*}, M(a)\right)$, i.e., the potential outcome for the terminal event time when the treatment is set to $a^{*}$ and the nonterminal event time is set to $M(a)$, the counterfactual nonterminal event time when the treatment is set to $a$. This quantity is a cross-world variable. In order to respect the semi-competing risk data structure, we need to restrict our attention to scenarios that if the nonterminal event ever happens, it happens before the terminal event, such that $T\left(a^{*}, M(a)\right)$ is well-defined. This is formally given as the following cross-world ordering invariance assumption:

Assumption 1. For any $a, a^{*} \in\{0,1\}$, either (i) $M(a)<\infty$ and $T\left(a^{*}, M(a)\right) \geq$ $M(a)$ or (ii) $M(a)=\infty$ and $T\left(a^{*}, M(a)\right)<M(a)$ holds.

Note that without Assumption $1, T\left(a^{*}, M(a)\right)$ may not be well-defined, and mediation analysis would be pointless.

Furthermore, the potential non-terminal event may or may not occur 
2.2 Counterfactuals and causal estimands

before the potential primary event time under different treatment assignments, which motivates us to examine causal effects based on our proposed principal stratification approach, extended from Frangakis and $\mathrm{Ru}-$ bin (2002). Intuitively, we stratify the study population into latent classes identified by $U$ with 3 categories based on whether they are susceptible to the nonterminal event under different treatment assignments:

1. $U=1: M(0) \leq T(0)$ and $M(1) \leq T(1)$ (always susceptible).

2. $U=2: M(0) \leq T(0)$ and $M(1)=\infty$ (prevented).

3. $U=3: M(0)=\infty$ and $M(1)=\infty$ (always non-susceptible).

Here, we do not have a fourth stratum: $M(0)=\infty$ and $M(1) \leq T(1)$, such that the treatment never convert a subject from non-susceptible to susceptible. This restriction is along the same line as the "no defier" assumption commonly adopted in the instrumental variables methods, suggesting that the treatment effect is "monotone" and no reversed effect for the subjects (Angrist et al., 1996).

Remark 1. The defined strata (and associated stratum-specific effects) are substantially different from the survivors' principal stratum (and the survivor average causal effect (SACE)) that is commonly defined in "truncation by death" literature (Zhang and Rubin, 2003, Comment et al., 2019). In 
2.2 Counterfactuals and causal estimands

particular, the survivors' principal stratum is defined by $\{T(0) \geq t, T(1) \geq$ $t$ \} for some fixed time $t$ in Comment et al. (2019), whereas our definition does not depend on any arbitrary post-treatment time $t$.

Remark 2. Lin et al. (2017) explained the difficulties in defining natural mediation effects in survival context with longitudinal mediators. They defined interventional effects in a discrete-time setting, where the mediators and past survival status are subject to a hypothetical intervention. They mentioned that principal stratification as an alternative framework to avoid such hypothetical intervention, but did not explore further. We consider a different setting that shares some of the difficulties, but also with unique data structure so that principal strata can be defined.

Under suitable assumptions (to be made clear in Section 2.3), for $U=1$, the joint distribution of $(T(a), M(a)), a=0,1$ could be nonparametrically identified on the upper wedge of the positive quadrant, and by cross-world invariance, $T(1, M(0)) \geq M(0)$ is well-defined in the same region, and therefore we can define and estimate the stratum-specific natural indirect and direct effects:

$$
\begin{aligned}
N I E_{1}(t ; \boldsymbol{x})= & \operatorname{Pr}\{T(1, M(1)) \geq t \mid \boldsymbol{X}=\boldsymbol{x}, U=1\} \\
& -\operatorname{Pr}\{T(1, M(0)) \geq t \mid \boldsymbol{X}=\boldsymbol{x}, U=1\}
\end{aligned}
$$


2.2 Counterfactuals and causal estimands

and

$$
\begin{aligned}
N D E_{1}(t ; \boldsymbol{x})= & \operatorname{Pr}\{T(1, M(0)) \geq t \mid \boldsymbol{X}=\boldsymbol{x}, U=1\} \\
& -\operatorname{Pr}\{T(0, M(0)) \geq t \mid \boldsymbol{X}=\boldsymbol{x}, U=1\} .
\end{aligned}
$$

In stratum with $U=2$, although the pair $(T(1, M(0)), M(0))$ is technically defined on the upper wedge, $(T(1), M(1))$ is not defined in that region as $M(1)=\infty$, hence there is no common support when the mediator is being considered and we do not seek to estimate the indirect and direct effects. However, the stratum-specific total effect can still be estimated:

$$
T E_{2}(t ; \boldsymbol{x})=\operatorname{Pr}\{T(1) \geq t \mid \boldsymbol{X}=\boldsymbol{x}, U=2\}-\operatorname{Pr}\{T(0) \geq t \mid \boldsymbol{X}=\boldsymbol{x}, U=2\} .
$$

In stratum with $U=3, M(0)=M(1)=\infty$ and $T(1, M(0))=T(1, M(1))$ so there is no indirect effect. The stratum-specific total effect can still be defined as

$$
T E_{3}(t ; \boldsymbol{x})=\operatorname{Pr}\{T(1) \geq t \mid \boldsymbol{X}=\boldsymbol{x}, U=3\}-\operatorname{Pr}\{T(0) \geq t \mid \boldsymbol{X}=\boldsymbol{x}, U=3\} .
$$

Remark 3. In principle, a mediator shall satisfy temporal precedence, that it shall occur before the primary event. Therefore, one can view that the mediator is technically absent in $U=3$, and an attempt to define mediation effects would be futile. In $U=2$, the presence of the mediator before the primary event only happens in one treatment level with certainty. As a 
result, one cannot fix the mediator level at a different treatment level, and mediation effects cannot be defined. Note that in $U=2, T E_{2}$ can be interpreted as the treatment effect in survival among individuals whose mediating events are prevented by the treatment.

\subsection{Identification}

To identify the stratum-specific natural indirect and direct effects and stratumspecific total effects, we impose the following assumptions.

Assumption 2. If $A=a$, then $M=M(a)$ and $T=T(a)$ with probability one.

Assumption 3. For $a, a^{*} \in\{0,1\}$ and $u \in\{1,2,3\}$,

$$
\left\{T\left(a, M\left(a^{*}\right)\right), M\left(a^{*}\right)\right\} \perp A \mid \boldsymbol{X}, U=u
$$

and

$$
\begin{aligned}
& \operatorname{Pr}\left(T\left(a, M\left(a^{*}\right)\right) \mid M\left(a^{*}\right)=m, A=a, \boldsymbol{X}, U=u\right) \\
& \quad=\operatorname{Pr}(T(a, M(a)) \mid M(a)=m, A=a, \boldsymbol{X}, U=u) .
\end{aligned}
$$

Assumptions 2 is the standard consistency assumption for causal inference. Assumption 3 serves a similar purpose as the sequential ignorability assumption (Imai et al. 2010) but is weaker so that the the assumption 
2.3 Identification

holds within a stratum and only requires $T\left(a, M\left(a^{*}\right)\right)$ to be well-defined. Based on Assumptions 2 - 3, we are able to connect the stratum-specific natural indirect and direct effects and stratum-specific total effects with the distribution of the observed data given stratum membership as follows.

Theorem 1. Under Assumptions 2- 3, for stratum with $U=1$, the stratum-specific natural indirect effect $N I E_{1}(t ; \boldsymbol{x})$ is equal to

$$
\begin{aligned}
& \int_{0}^{t}\{1-\operatorname{Pr}(T<t \mid M=m, \boldsymbol{X}=\boldsymbol{x}, A=1, U=1)\} \\
& \quad \times\left\{d F_{M \mid \boldsymbol{X}=\boldsymbol{x}, A=1, U=1}(m)-d F_{M \mid \boldsymbol{X}=\boldsymbol{x}, A=0, U=1}(m)\right\} \\
& \quad+\operatorname{Pr}(M \leq t \mid \boldsymbol{X}=\boldsymbol{x}, A=0, U=1)-\operatorname{Pr}(M \leq t \mid \boldsymbol{X}=\boldsymbol{x}, A=1, U=1)
\end{aligned}
$$

and the stratum-specific natural direct effect $N D E_{1}(t ; \boldsymbol{x})$ is equal to

$$
\begin{aligned}
& \int_{0}^{t}\{\operatorname{Pr}(T<t \mid M=m, \boldsymbol{X}=\boldsymbol{x}, A=0, U=1) \\
& \quad-\operatorname{Pr}(T<t \mid M=m, \boldsymbol{X}=\boldsymbol{x}, A=1, U=1)\} d F_{M \mid \boldsymbol{X}=\boldsymbol{x}, A=0, U=1}(m) .
\end{aligned}
$$

Under Assumptions 2 and 3, for stratum with $U=2$, the stratum-specific total effect $T E_{2}(t ; \boldsymbol{x})$ is equal to

$$
\operatorname{Pr}(T \geq t \mid A=1, \boldsymbol{X}=\boldsymbol{x}, U=2)-\operatorname{Pr}(T \geq t \mid A=0, \boldsymbol{X}=\boldsymbol{x}, U=2)
$$

and for stratum with $U=3$, the stratum-specific total effect $T E_{3}(t ; \boldsymbol{x})$ is equal to

$$
\operatorname{Pr}(T \geq t \mid A=1, \boldsymbol{X}=\boldsymbol{x}, U=3)-\operatorname{Pr}(T \geq t \mid A=0, \boldsymbol{X}=\boldsymbol{x}, U=3)
$$


The proof of Theorem 1 is given in Section S1.1 of the online supplement. Since $U$ is unobserved, we cannot use Theorem 1 directly to identify those stratum-specific effects from observed data. To do so, one would further assume

Assumption 4. With probability one, $U$ is conditional independent of $A$ given $\boldsymbol{X}$.

Assumption 5. With probability one,

$$
\begin{aligned}
& \operatorname{Pr}(M(0)=m \mid \boldsymbol{X}=\boldsymbol{x}, U=2)=g_{1}\{\operatorname{Pr}(M(0)=m \mid \boldsymbol{X}=\boldsymbol{x}, U=1) ; \boldsymbol{x}\}, \\
& \operatorname{Pr}(T(0) \geq t \mid M(0)=m, \boldsymbol{X}=\boldsymbol{x}, U=2) \\
& \quad=g_{2}\{\operatorname{Pr}(T(0) \geq t \mid M(0)=m, \boldsymbol{X}=\boldsymbol{x}, U=1) ; \boldsymbol{x}\}, \\
& \operatorname{Pr}(T(1) \geq t \mid \boldsymbol{X}=\boldsymbol{x}, U=2)=g_{3}\{\operatorname{Pr}(T(1) \geq t \mid \boldsymbol{X}=\boldsymbol{x}, U=3) ; \boldsymbol{x}\},
\end{aligned}
$$

for some known functions $g_{k}(\cdot ; \boldsymbol{x})(k=1,2,3)$ that satisfies conditions given in Section S1.2 of the online supplement.

Assumption 6. $(M, T, U)$ is conditionally independent of $C$ given $A$ and $\boldsymbol{X}$, and the upper bound of the support of $T$ is no larger than that of $C$.

Assumption 4 requires that the stratum membership is not affected by the treatment assignment $A$ given covariates $\boldsymbol{X}$. Assumption 5 requires a known relationship of stratum-specific event time distributions that guarantees the identification of the stratum-specific distributions. It may be 
relaxed by assuming unknown $g_{k}$ 's that can be parameterized and estimated with the observed data. For example, we may assume proportional hazards models for the stratum-specific event times as in Section 2.4. The first part of Assumption 6 is a standard assumption for non-informative censoring time. The second part of Assumption 6 is an extension of the independent censoring and sufficient follow-up assumption in Maller and Zhou (1992) for nonparametric estimation of cured proportion in censored data. The assumption on the upper bounds of the supports ensures sufficient observation of the tail behavior of the event times for identification of stratum membership. By further assuming Assumptions 4- 6, we obtain the identification results in Theorem 2, whose proof is given in Section S1.2 of the online supplement.

Theorem 2. Under Assumptions [- 6, the stratum-specific effects can be identified, with identification formulas given in Section S1.2 of the online supplement.

Theorem 2 gives the identification result based on nonparametric models for $U$ and for $(M, T)$ given $U$. In particular, Assumption 4 requires some modeling assumptions to be made. In practice, we may consider additional model assumptions for $U$ and $(M, T)$ to gain power in understanding the causal effects. In the next section, we extend the multistate modeling idea 
2.4 Modeling assumptions

in the literature of semi-competing risks data to form such a model.

\subsection{Modeling assumptions}

One way to model semi-competing risks data is to use a multistate framework $\mathrm{Xu}$ et al., 2010). In multistate analysis of semi-competing risks data, usually three states are involved, corresponding to healthy (state 1), illness (state 2), and death (state 3) in an illness-death model. All subjects starts

at state 1 . A subject enters state 2 if he/she develops the intermediate event, while he/she enters state 3 if he/she develops the primary event. In traditional illness-death model for semi-competing risks data, three processes moving from one state to another are modeled: (1) healthy to illness, (2) illness to death, and (3) healthy to death.

Here, we extend the idea and model the processes moving from one state to another in different strata defined in Section 2.2. For subjects with $U=1$ and subjects with $U=2$ receiving $A=0$, the processes of healthy to illness and illness to death are involved and we model the time to the nonterminal event $M$ and the residual time $R \equiv T-M$. We assume that $M$ and $R$ are conditionally independent given $A, \boldsymbol{X}$, and $U$. This serves two purposes: to obtain a tractable EM algorithm, and to avoid the problem of induced informative censoring caused by residual dependence between $M$ 
2.4 Modeling assumptions and $R$ (Wang and Wells, 1998; Lin et al., 1999). For subjects with $U=2$ receiving $A=1$ and subjects with $U=3$, the process of healthy to death is involved. This proposed model is related to but different from the illnessdeath model, in that subjects in different principal strata may experience different transition structure in our proposed model.

Suppose that for a subject with $U=1$, the nonterminal event time follows a proportional hazards model with hazard function given by

$$
\lambda_{M}^{(1)}(t \mid A=a, \boldsymbol{X}=\boldsymbol{x})=\lambda_{1}(t) \exp \left(\beta_{M 1} a+\boldsymbol{\gamma}_{M 1}^{\mathrm{T}} \boldsymbol{x}\right)
$$

and the gap time between the occurrences of nonterminal and terminal events $R$ follows a proportional hazards model with hazard function given by

$$
\lambda_{R}^{(1)}(r \mid A=a, \boldsymbol{X}=\boldsymbol{x})=\lambda_{2}(r) \exp \left(\beta_{R 1} a+\boldsymbol{\gamma}_{R 1}^{\mathrm{T}} \boldsymbol{x}\right)
$$

Suppose that for subject with $U=2$ and unexposed to treatment $(A=$ 0 ), the nonterminal event time follows a proportional hazards model with hazard function given by

$$
\lambda_{M}^{(2)}(t \mid A=0, \boldsymbol{X}=\boldsymbol{x})=\lambda_{1}(t) \exp \left(\beta_{M 2}+\gamma_{M 2}^{\mathrm{T}} \boldsymbol{x}\right),
$$

and the gap time between the occurrences of nonterminal and terminal events follows another proportional hazards model with hazard function 
given by

$$
\lambda_{R}^{(2)}(r \mid A=0, \boldsymbol{X}=\boldsymbol{x})=\lambda_{2}(r) \exp \left(\beta_{R 2}+\gamma_{R 2}^{\mathrm{T}} \boldsymbol{x}\right) .
$$

Here, subjects with $U=1$ and subjects with $U=2$ unexposed to treatment share the same baseline hazard functions, although the hazard ratios for covariates may be different. The parameters $\beta_{M 1}$ and $\beta_{R 1}$ are the log hazard ratios of treatment on the nonterminal event time and gap time, respectively, for subjects with $U=1$; the parameters $\beta_{M 2}$ and $\beta_{R 2}$ are the log hazard ratios on the nonterminal event time and gap time, respectively, comparing subjects with $U=1$ and $U=2$ who both unexposed to treatment with baseline covariates value $\boldsymbol{X}=\mathbf{0}$.

For subject with $U=2$ and exposed with treatment $(A=1)$, we assume that the terminal event time follows a proportional hazards model with hazard function given by

$$
\lambda_{T}^{(2)}(t \mid A=1, \boldsymbol{X}=\boldsymbol{x})=\lambda_{3}(t) \exp \left(\beta_{T 2}+\boldsymbol{\gamma}_{T 2}^{\mathrm{T}} \boldsymbol{x}\right)
$$

For subject with $U=3$, we suppose that the terminal event time follows a proportional hazards model with hazard function given by

$$
\lambda_{T}^{(3)}(t \mid A=a, \boldsymbol{X}=\boldsymbol{x})=\lambda_{3}(t) \exp \left(\beta_{T 3} a+\boldsymbol{\gamma}_{T 3}^{\mathrm{T}} \boldsymbol{x}\right) .
$$

Note that the terminal event times for subject with $U=3$ and subject with $U=2$ exposed to treatment share the same baseline hazard function. 
2.4 Modeling assumptions

The parameter $\beta_{T 3}$ is the log hazard ratio of treatment on the terminal event time for subjects with $U=3$, while $\beta_{T 2}$ is the log hazard ratio of the terminal event time comparing subjects with $U=3$ and $A=0$ with subjects with $U=2$ and $A=1$, with the same covariates value $\boldsymbol{X}=\mathbf{0}$.

Making use of the results in Theorem 1, we obtain the expression of the stratum-specific effects by replacing the probabilities by the expressions under the proportional hazards models. The natural indirect and direct effects in stratum with $U=1$ can be presented as

$$
\begin{aligned}
& N I E_{1}(t \mid \boldsymbol{X}=\boldsymbol{x})=\int_{0}^{t} \exp \left\{-\Lambda_{2}(t-m) e^{\beta_{R 1}+\boldsymbol{\gamma}_{R 1}^{\mathrm{T}} \boldsymbol{x}}\right\} \lambda_{1}(m) e^{\boldsymbol{\gamma}_{M 1}^{\mathrm{T}} \boldsymbol{x}} \\
& \quad \times\left[e^{\beta_{M 1}} \exp \left\{-\Lambda_{1}(m) e^{\beta_{M 1}+\boldsymbol{\gamma}_{M 1}^{\mathrm{T}} \boldsymbol{x}}\right\}-\exp \left\{-\Lambda_{1}(m) e^{\boldsymbol{\gamma}_{M 1}^{\mathrm{T}} \boldsymbol{x}}\right\}\right] d m \\
& \quad+\exp \left\{-\Lambda_{1}(t) e^{\beta_{M 1}+\boldsymbol{\gamma}_{M 1}^{\mathrm{T}} \boldsymbol{x}}\right\}-\exp \left\{-\Lambda_{1}(t) e^{\boldsymbol{\gamma}_{M 1}^{\mathrm{T}} \boldsymbol{x}}\right\}
\end{aligned}
$$

and

$$
\begin{aligned}
N D E_{1}(t \mid \boldsymbol{X}=\boldsymbol{x})=\int_{0}^{t} & {\left[\exp \left\{-\Lambda_{2}(t-m) e^{\beta_{R 1}+\boldsymbol{\gamma}_{R 1}^{\mathrm{T}} \boldsymbol{x}}\right\}-\exp \left\{-\Lambda_{2}(t-m) e^{\boldsymbol{\gamma}_{R 1}^{\mathrm{T}} \boldsymbol{x}}\right\}\right] } \\
& \times \lambda_{1}(m) e^{\boldsymbol{\gamma}_{M 1}^{\mathrm{T}} \boldsymbol{x}} \exp \left\{-\Lambda_{1}(m) e^{\boldsymbol{\gamma}_{M 1}^{\mathrm{T}} \boldsymbol{x}}\right\} d m,
\end{aligned}
$$

where $\Lambda_{1}(t)=\int_{0}^{t} \lambda_{1}(s) d s$ and $\Lambda_{2}(t)=\int_{0}^{t} \lambda_{2}(s) d s$. The total effects in strata with $U=2$ and $U=3$ are given by

$$
\begin{aligned}
& T E_{2}(t \mid \boldsymbol{X}=\boldsymbol{x})=\exp \left\{-\Lambda_{3}(t) e^{\beta_{T 2}+\boldsymbol{\gamma}_{T 2}^{\mathrm{T}} \boldsymbol{x}}\right\}-1+\int_{0}^{t} \lambda_{1}(m) e^{\beta_{M 2}+\boldsymbol{\gamma}_{M 2}^{\mathrm{T}} \boldsymbol{x}} \\
& \times \exp \left\{-\Lambda_{1}(m) e^{\beta_{M 2}+\boldsymbol{\gamma}_{M 2}^{\mathrm{T}} \boldsymbol{x}}\right\}\left[1-\exp \left\{-\Lambda_{2}(t-m) e^{\beta_{R 2}+\boldsymbol{\gamma}_{R 2}^{\mathrm{T}} \boldsymbol{x}}\right\}\right] d m
\end{aligned}
$$


and

$$
T E_{3}(t \mid \boldsymbol{X}=\boldsymbol{x})=\exp \left\{-\Lambda_{3}(t) e^{\beta_{T 3}+\boldsymbol{\gamma}_{T 3}^{\mathrm{T}} \boldsymbol{x}}\right\}-\exp \left\{-\Lambda_{3}(t) e^{\boldsymbol{\gamma}_{T 3}^{\mathrm{T}} \boldsymbol{x}}\right\}
$$

where $\Lambda_{3}(t)=\int_{0}^{t} \lambda_{3}(s) d s$.

As in $\mathrm{Yu}$ et al. (2015), we consider a multinomial logistic regression model on the stratum membership. In particular, we assume

$$
\begin{aligned}
& w_{1}(\boldsymbol{x} ; \boldsymbol{\alpha})=\operatorname{Pr}(U=1 \mid \boldsymbol{X}=\boldsymbol{x})=\frac{\exp \left(\boldsymbol{\alpha}_{1}^{\mathrm{T}} \widetilde{\boldsymbol{x}}\right)}{1+\exp \left(\boldsymbol{\alpha}_{1}^{\mathrm{T}} \widetilde{\boldsymbol{x}}\right)+\exp \left(\boldsymbol{\alpha}_{2}^{\mathrm{T}} \widetilde{\boldsymbol{x}}\right)}, \\
& w_{2}(\boldsymbol{x} ; \boldsymbol{\alpha})=\operatorname{Pr}(U=2 \mid \boldsymbol{X}=\boldsymbol{x})=\frac{\exp \left(\boldsymbol{\alpha}_{2}^{\mathrm{T}} \widetilde{\boldsymbol{x}}\right)}{1+\exp \left(\boldsymbol{\alpha}_{1}^{\mathrm{T}} \widetilde{\boldsymbol{x}}\right)+\exp \left(\boldsymbol{\alpha}_{2}^{\mathrm{T}} \widetilde{\boldsymbol{x}}\right)},
\end{aligned}
$$

and $w_{3}(\boldsymbol{x} ; \boldsymbol{\alpha})=\operatorname{Pr}(U=3 \mid \boldsymbol{X}=\boldsymbol{x})=\left\{1+\exp \left(\boldsymbol{\alpha}_{1}^{\mathrm{T}} \widetilde{\boldsymbol{x}}\right)+\exp \left(\boldsymbol{\alpha}_{2}^{\mathrm{T}} \widetilde{\boldsymbol{x}}\right)\right\}^{-1}$, where $\boldsymbol{\alpha}=\left(\boldsymbol{\alpha}_{1}^{\mathrm{T}}, \boldsymbol{\alpha}_{2}^{\mathrm{T}}\right)^{\mathrm{T}}$ and $\widetilde{\boldsymbol{x}}=\left(1, \boldsymbol{x}^{\mathrm{T}}\right)^{\mathrm{T}}$. Then, the marginalized stratumspecific natural indirect and direct effects are given by

$$
N I E_{1}(t)=\frac{\int N I E_{1}(t \mid \boldsymbol{X}=\boldsymbol{x}) w_{1}(\boldsymbol{x} ; \boldsymbol{\alpha}) d F(\boldsymbol{x})}{\int w_{1}(\boldsymbol{x} ; \boldsymbol{\alpha}) d F(\boldsymbol{x})}
$$

and

$$
N D E_{1}(t)=\frac{\int N D E_{1}(t \mid \boldsymbol{X}=\boldsymbol{x}) w_{1}(\boldsymbol{x} ; \boldsymbol{\alpha}) d F(\boldsymbol{x})}{\int w_{1}(\boldsymbol{x} ; \boldsymbol{\alpha}) d F(\boldsymbol{x})}
$$

where $F(\cdot)$ is the cumulative distribution function of $\boldsymbol{X}$.

\subsection{Nonparametric maximum likelihood estimation}

For a random sample of $n$ subjects, the observed semi-competing risks data are given by $\mathcal{O}=\left\{\mathcal{O}_{i}: i=1, \ldots, n\right\}$, where $\mathcal{O}_{i}=\left\{\Delta_{i}^{M}, Z_{i}, \Delta_{i}^{T}, Y_{i}, A_{i}, \boldsymbol{X}_{i}\right\}$. 
2.5 Nonparametric maximum likelihood estimation

The likelihood function for the observed data can be constructed by laying out the conditional probabilities of the observed data given possible stratum membership. Specifically, if $\Delta_{i}^{M}=1$, then $U_{i}=3$ with probability zero, and $U_{i}=1$ with probability one if further $A_{i}=1$. If $\Delta_{i}^{M}=0$ and $\Delta_{i}^{T}=1$, then $U_{i}=1$ with probability zero, and $U_{i}=3$ with probability one if further $A_{i}=0$. If $\Delta_{i}^{M}=\Delta_{i}^{T}=0$, then there are positive probabilities for $U_{i}=1,2,3$. The likelihood function for the observed data $\mathcal{O}$ is then given by

$$
\prod_{i=1}^{n} \widetilde{L}_{i 1}\left(\mathcal{O}_{i}\right)^{\Delta_{i}^{M}}\left\{\widetilde{L}_{i 2}\left(\mathcal{O}_{i}\right)^{\Delta_{i}^{T}} \widetilde{L}_{i 3}\left(\mathcal{O}_{i}\right)^{1-\Delta_{i}^{T}}\right\}^{1-\Delta_{i}^{M}}
$$

where

$$
\begin{aligned}
\widetilde{L}_{i 1}\left(\mathcal{O}_{i}\right)= & \operatorname{Pr}\left(U_{i}=1 \mid \boldsymbol{X}_{i}\right) \operatorname{Pr}\left(Z_{i}, Y_{i}, \Delta_{i}^{T} \mid U_{i}=1, \boldsymbol{X}_{i}, A_{i}\right) \\
& +I\left(A_{i}=0\right) \operatorname{Pr}\left(U_{i}=2 \mid \boldsymbol{X}_{i}\right) \operatorname{Pr}\left(Z_{i}, Y_{i}, \Delta_{i}^{T} \mid U_{i}=2, \boldsymbol{X}_{i}, A_{i}=0\right), \\
\widetilde{L}_{i 2}\left(\mathcal{O}_{i}\right)= & \operatorname{Pr}\left(U_{i}=3 \mid \boldsymbol{X}_{i}\right) \operatorname{Pr}\left(Y_{i}, \Delta_{i}^{T} \mid U_{i}=3, \boldsymbol{X}_{i}\right) \\
& +I\left(A_{i}=1\right) \operatorname{Pr}\left(U_{i}=2 \mid \boldsymbol{X}_{i}\right) \operatorname{Pr}\left(Y_{i}, \Delta_{i}^{T} \mid U_{i}=2, \boldsymbol{X}_{i}, A_{i}=1\right),
\end{aligned}
$$

and

$$
\begin{aligned}
\widetilde{L}_{i 3}\left(\mathcal{O}_{i}\right)= & \operatorname{Pr}\left(U_{i}=1 \mid \boldsymbol{X}_{i}\right) \operatorname{Pr}\left(Z_{i}, \Delta_{i}^{M}, Y_{i}, \Delta_{i}^{T} \mid U_{i}=1, \boldsymbol{X}_{i}\right) \\
+ & \operatorname{Pr}\left(U_{i}=2 \mid \boldsymbol{X}_{i}\right)\left\{I\left(A_{i}=0\right) \operatorname{Pr}\left(Z_{i}, \Delta_{i}^{M}, Y_{i}, \Delta_{i}^{T} \mid U_{i}=2, \boldsymbol{X}_{i}, A_{i}=0\right)\right. \\
& \left.+I\left(A_{i}=1\right) \operatorname{Pr}\left(Y_{i}, \Delta_{i}^{T} \mid U_{i}=2, \boldsymbol{X}_{i}, A_{i}=1\right)\right\}
\end{aligned}
$$




\subsection{Nonparametric maximum likelihood estimation}

$$
+\operatorname{Pr}\left(U_{i}=3 \mid \boldsymbol{X}_{i}\right) \operatorname{Pr}\left(Y_{i}, \Delta_{i}^{T} \mid U_{i}=3, \boldsymbol{X}_{i}\right) .
$$

We consider the nonparametric maximum likelihood estimation such that the estimators for $\Lambda_{1}, \Lambda_{2}$, and $\Lambda_{3}$ are step functions, as in Zeng and Lin (2007). In particular, let $0<t_{11}<\cdots<t_{1 m_{1}}<\infty$ be the ordered sequence of event times $Z_{i}$ 's with $\Delta_{i}^{M}=1$; let $0<t_{21}<\cdots<t_{2 m_{2}}<\infty$ be the ordered sequence of gap times $V_{i} \equiv Y_{i}-Z_{i}$ 's with $\Delta_{i}^{M}=\Delta_{i}^{T}=1$; and let $0<t_{31}<\cdots<t_{3 m_{3}}<\infty$ be the ordered sequence of event times $Y_{i}^{\prime}$ 's with $\Delta_{i}^{M}=0$ and $\Delta_{i}^{T}=1$. Let $\lambda_{k l}$ be the jump size for $\Lambda_{k}$ at $t_{k l}$ for $k=1,2,3$ and $l=1, \ldots, m_{k}$. Write $\boldsymbol{\eta}_{M 1}=\left(\beta_{M 1}, \gamma_{M 1}\right)^{\mathrm{T}}, \boldsymbol{\eta}_{R 1}=\left(\beta_{R 1}, \boldsymbol{\gamma}_{R 1}\right)^{\mathrm{T}}, \boldsymbol{\eta}_{M 2}=$ $\left(\beta_{M 2}, \boldsymbol{\gamma}_{M 2}\right)^{\mathrm{T}}, \boldsymbol{\eta}_{R 2}=\left(\beta_{R 2}, \boldsymbol{\gamma}_{R 2}\right)^{\mathrm{T}}, \boldsymbol{\eta}_{T 2}=\left(\beta_{T 2}, \boldsymbol{\gamma}_{T 2}\right)^{\mathrm{T}}, \boldsymbol{\eta}_{T 3}=\left(\beta_{T 3}, \boldsymbol{\gamma}_{T 3}\right)^{\mathrm{T}}$, $\boldsymbol{\theta}=\left(\boldsymbol{\eta}_{M 1}^{\mathrm{T}}, \boldsymbol{\eta}_{R 1}^{\mathrm{T}}, \boldsymbol{\eta}_{M 2}^{\mathrm{T}}, \boldsymbol{\eta}_{R 2}^{\mathrm{T}}, \boldsymbol{\eta}_{T 2}^{\mathrm{T}}, \boldsymbol{\eta}_{T 3}^{\mathrm{T}}, \boldsymbol{\alpha}^{\mathrm{T}}\right)^{\mathrm{T}}$, and $\mathcal{A}=\left(\Lambda_{1}, \Lambda_{2}, \Lambda_{3}\right)^{\mathrm{T}}$. We maximize the objective function

$$
L_{n}(\boldsymbol{\theta}, \mathcal{A})=\prod_{i=1}^{n} L_{i 1}(\boldsymbol{\theta}, \mathcal{A})^{\Delta_{i}^{M}}\left\{L_{i 2}(\boldsymbol{\theta}, \mathcal{A})^{\Delta_{i}^{T}} L_{i 3}(\boldsymbol{\theta}, \mathcal{A})^{1-\Delta_{i}^{T}}\right\}^{1-\Delta_{i}^{M}},
$$

where

$$
\begin{aligned}
L_{i 1}(\boldsymbol{\theta}, \mathcal{A})= & w_{1}\left(\boldsymbol{X}_{i} ; \boldsymbol{\alpha}\right) \Lambda_{1}\left\{Z_{i}\right\} e^{\boldsymbol{\eta}_{M 1}^{\mathrm{T}} \boldsymbol{W}_{i}} \exp \left(-e^{\boldsymbol{\eta}_{M 1}^{\mathrm{T}} \boldsymbol{W}_{i}} \sum_{t_{1 l} \leq Z_{i}} \lambda_{1 l}\right) \\
& \times\left(\Lambda_{2}\left\{V_{i}\right\} e^{\boldsymbol{\eta}_{R 1}^{\mathrm{T}} \boldsymbol{W}_{i}}\right)^{\Delta_{i}^{T}} \exp \left(-e^{\boldsymbol{\eta}_{R 1}^{\mathrm{T}} \boldsymbol{W}_{i}} \sum_{t_{2 l} \leq V_{i}} \lambda_{2 l}\right) \\
& +I\left(A_{i}=0\right) w_{2}\left(\boldsymbol{X}_{i} ; \boldsymbol{\alpha}\right) \Lambda_{1}\left\{Z_{i}\right\} e^{\boldsymbol{\eta}_{M 2}^{\mathrm{T}} \widetilde{\boldsymbol{X}}_{i}} \exp \left(-e^{\boldsymbol{\eta}_{M 2}^{\mathrm{T}} \widetilde{\boldsymbol{X}}_{i}} \sum_{t_{1 l} \leq Z_{i}} \lambda_{1 l}\right)
\end{aligned}
$$


2.5 Nonparametric maximum likelihood estimation

$$
\begin{gathered}
\times\left(\Lambda_{2}\left\{V_{i}\right\} e^{\boldsymbol{\eta}_{R 2}^{\mathrm{T}} \widetilde{\boldsymbol{X}}_{i}}\right)^{\Delta_{i}^{T}} \exp \left(-e^{\boldsymbol{\eta}_{R 2}^{\mathrm{T}} \widetilde{\boldsymbol{X}}_{i}} \sum_{t_{2 l} \leq V_{i}} \lambda_{2 l}\right) \\
L_{i 2}(\boldsymbol{\theta}, \mathcal{A})=I\left(A_{i}=1\right) w_{2}\left(\boldsymbol{X}_{i} ; \boldsymbol{\alpha}\right)\left(\Lambda_{3}\left\{Y_{i}\right\} e^{\boldsymbol{\eta}_{T 2}^{\mathrm{T}} \widetilde{\boldsymbol{X}}_{i}}\right)^{\Delta_{i}^{T}} \exp \left(-\sum_{t_{3 l} \leq Y_{i}} \lambda_{3 l} e^{\boldsymbol{\eta}_{T 2}^{\mathrm{T}} \widetilde{\boldsymbol{X}}_{i}}\right) \\
+w_{3}\left(\boldsymbol{X}_{i} ; \boldsymbol{\alpha}\right)\left(\Lambda_{3}\left\{Y_{i}\right\} e^{\boldsymbol{\eta}_{T 3}^{\mathrm{T}} \boldsymbol{W}_{i}}\right)^{\Delta_{i}^{T}} \exp \left(-\sum_{t_{3 l} \leq Y_{i}} \lambda_{3 l} \boldsymbol{\eta}^{\boldsymbol{\eta}_{T 3}^{\mathrm{T}} \boldsymbol{W}_{i}}\right) \\
L_{i 3}(\boldsymbol{\theta}, \mathcal{A})=L_{i 2}(\boldsymbol{\eta}, \mathcal{A})+w_{1}\left(\boldsymbol{X}_{i} ; \boldsymbol{\alpha}\right) \exp \left(-\sum_{t_{1 l} \leq Z_{i}} \lambda_{1 l} e^{\boldsymbol{\eta}_{M 1}^{\mathrm{T}} \boldsymbol{W}_{i}}\right) \\
+I\left(A_{i}=0\right) w_{2}\left(\boldsymbol{X}_{i} ; \boldsymbol{\alpha}\right) \exp \left(-\sum_{t_{1 l} \leq Z_{i}} \lambda_{1 l} e^{\boldsymbol{\eta}_{M 2}^{\mathrm{T}} \widetilde{\boldsymbol{X}}_{i}}\right)
\end{gathered}
$$

$\boldsymbol{W}_{i}=\left(A_{i}, \boldsymbol{X}_{i}^{\mathrm{T}}\right)^{\mathrm{T}}$, and $\Lambda_{k}\{t\}$ is the jump size of $\Lambda_{k}$ at time $t$ for $k=1,2,3$.

By treating $U_{i}(i=1, \ldots, n)$ as missing data, we propose an EM algorithm to maximize this objective function. In the E-step of the EM algorithm, we evaluate the conditional expectation of terms related to the latent variable $U_{i}$ that follows a multinomial distribution with probabilities corresponding to the observed-data likelihood given different values of $U_{i}$. In the M-step, we update the parameter values by partial-score-structured estimating equations, Breslow-type estimators, and logistic-regression-type estimating equations. The details of the EM algorithm are given in Section $\mathrm{S} 2$ of the online supplement. We write $(\widehat{\boldsymbol{\theta}}, \widehat{\mathcal{A}})$ as the estimators. The indirect and direct effects in stratum with $U=1$ can then be estimated by

$$
\widehat{N I E}_{1}(t ; \boldsymbol{x})=\sum_{t_{1 j} \leq t}\left[\exp \left(-\sum_{t_{2 k} \leq t-t_{1 j}} \widehat{\lambda}_{2 k} \widehat{\widehat{\boldsymbol{e}}}^{\mathrm{T} \mathrm{T}} \widetilde{\boldsymbol{x}}\right) \widehat{\lambda}_{1 j}\right.
$$


2.5 Nonparametric maximum likelihood estimation

$$
\begin{aligned}
& \left.\times\left\{e^{\widehat{\boldsymbol{\theta}}_{M 1}^{\mathrm{T}} \widetilde{\boldsymbol{x}}} \exp \left(-\sum_{k=1}^{j} \widehat{\lambda}_{1 k} e^{\widehat{\boldsymbol{\theta}}_{M 1}^{\mathrm{T}} \widetilde{\boldsymbol{x}}}\right)-e^{\widehat{\boldsymbol{\gamma}}_{M 1}^{\mathrm{T}} \boldsymbol{x}} \exp \left(-\sum_{k=1}^{j} \widehat{\lambda}_{1 k} e^{\widehat{\boldsymbol{\gamma}}_{M 1}^{\mathrm{T}} \boldsymbol{x}}\right)\right\}\right] \\
& +\exp \left(-\sum_{t_{1 j} \leq t} \widehat{\lambda}_{1 j} e^{\widehat{\boldsymbol{\theta}}_{M 1}^{\mathrm{T}} \widetilde{\boldsymbol{x}}}\right)-\exp \left(-\sum_{t_{1 j} \leq t} \widehat{\lambda}_{1 j} e^{\widehat{\boldsymbol{\gamma}}_{M 1}^{\mathrm{T}} \boldsymbol{x}}\right)
\end{aligned}
$$

and

$$
\begin{aligned}
& \widehat{N D E}_{1}(t ; \boldsymbol{x}) \\
=\sum_{t_{1 j} \leq t} & {\left[\left\{\exp \left(-\sum_{t_{2 k} \leq t-t_{1 j}} \widehat{\lambda}_{2 k} e^{\widehat{\boldsymbol{\theta}}_{R 1}^{\mathrm{T}} \widetilde{\boldsymbol{x}}}\right)-\exp \left(-\sum_{t_{2 k} \leq t-t_{1 j}} \widehat{\lambda}_{2 k} e^{\widehat{\boldsymbol{\gamma}}_{R 1}^{\mathrm{T}} \boldsymbol{x}}\right)\right\}\right.} \\
& \left.\times \widehat{\lambda}_{1 j} e^{\widehat{\boldsymbol{\gamma}}_{M 1}^{\mathrm{T}} \boldsymbol{x}} \exp \left(-\sum_{k=1}^{j} \widehat{\lambda}_{1 k} e^{\widehat{\boldsymbol{\gamma}}_{M 1}^{\mathrm{T}} \boldsymbol{x}}\right)\right]
\end{aligned}
$$

The total effects in strata with $U=2$ and $U=3$ can be estimated by

$$
\begin{aligned}
& \widehat{T E}_{2}(t ; \boldsymbol{x})=\exp \left(-\sum_{t_{3 j} \leq t} \widehat{\lambda}_{3 j} e^{\widehat{\boldsymbol{\theta}}_{T 2}^{\mathrm{T}} \widetilde{\boldsymbol{x}}}\right)-1 \\
& +\sum_{t_{1 j} \leq t}\left[\widehat{\lambda}_{1 j} e^{\widehat{\boldsymbol{\theta}}_{M 2}^{\mathrm{T}} \widetilde{\boldsymbol{x}}} \exp \left(-\sum_{k=1}^{j} \widehat{\lambda}_{1 k} e^{\widehat{\boldsymbol{\theta}}_{M 2}^{\mathrm{T}} \widetilde{\boldsymbol{x}}}\right)\left\{1-\exp \left(-\sum_{t_{2 k} \leq t-t_{1 j}} \widehat{\lambda}_{2 k} e^{\widehat{\boldsymbol{\theta}}_{R 2}^{\mathrm{T}} \widetilde{\boldsymbol{x}}}\right)\right\}\right]
\end{aligned}
$$

and

$$
\widehat{T E}_{3}(t ; \boldsymbol{x})=\exp \left(-\sum_{t_{3 j} \leq t} \widehat{\lambda}_{3 j} e^{\widehat{\boldsymbol{\theta}}_{T 3}^{\mathrm{T}} \widetilde{\boldsymbol{x}}}\right)-\exp \left(-\sum_{t_{3 j} \leq t} \widehat{\lambda}_{3 j} e^{\widehat{\boldsymbol{\gamma}}_{T 3}^{\mathrm{T}} \boldsymbol{x}}\right)
$$

The marginalized stratum-specific indirect and direct effects in stratum with $U=1$ can be estimated by

$$
\widehat{N I E}_{1}(t)=\frac{\sum_{i=1}^{n} w_{1}\left(\boldsymbol{X}_{i} ; \widehat{\boldsymbol{\alpha}}\right) \widehat{N I E}_{1}\left(t ; \boldsymbol{X}_{i}\right)}{\sum_{i=1}^{n} w_{1}\left(\boldsymbol{X}_{i} ; \widehat{\boldsymbol{\alpha}}\right)}
$$


and

$$
\widehat{N D E}_{1}(t)=\frac{\sum_{i=1}^{n} w_{1}\left(\boldsymbol{X}_{i} ; \widehat{\boldsymbol{\alpha}}\right) \widehat{N D E}_{1}\left(t ; \boldsymbol{X}_{i}\right)}{\sum_{i=1}^{n} w_{1}\left(\boldsymbol{X}_{i} ; \widehat{\boldsymbol{\alpha}}\right)}
$$

respectively.

\subsection{Asymptotic properties}

We study the asymptotic properties of the estimators under the semiparametric model in Section 2.4. Under suitable regularity conditions, the estimators $(\widehat{\boldsymbol{\theta}}, \widehat{\mathcal{A}})$ has the usual large sample properties, including consistency and asymptotic normality, as given in Theorem 3 below. Let $\boldsymbol{\theta}_{0}, \Lambda_{10}, \Lambda_{20}$, and $\Lambda_{30}$ be the true values of $\boldsymbol{\theta}, \Lambda_{1}, \Lambda_{2}$, and $\Lambda_{3}$, respectively, $\|\cdot\|$ be the Euclidean norm, and $\tau_{k}$ be the upper limit of the support of $\widehat{\Lambda}_{k}$ for $k=1,2,3$.

Theorem 3. Under Conditions 1-5 in Section S3 of the online supplement,

$$
\left\|\widehat{\boldsymbol{\theta}}-\boldsymbol{\theta}_{0}\right\|+\sum_{k=1}^{3} \sup _{t \in\left[0, \tau_{k}\right]}\left|\widehat{\Lambda}_{k}(t)-\Lambda_{k 0}(t)\right|
$$

converges to zero almost surely. In addition, $\sqrt{n}\left\{\widehat{\boldsymbol{\theta}}-\boldsymbol{\theta}_{0}, \widehat{\Lambda}_{1}(\cdot)-\Lambda_{10}(\cdot), \widehat{\Lambda}_{2}(\cdot)-\right.$ $\left.\Lambda_{20}(\cdot), \widehat{\Lambda}_{3}(\cdot)-\Lambda_{30}(\cdot)\right\}$ converges weakly to a zero-mean Gaussian process in the Banach space $\mathbb{R}^{m} \times l^{\infty}\left(\mathcal{A}_{1}\right) \times l^{\infty}\left(\mathcal{A}_{2}\right) \times l^{\infty}\left(\mathcal{A}_{3}\right)$, where $m$ is the dimension of $\boldsymbol{\theta}$ and $\mathcal{A}_{k}$ is the unit ball in the space of functions on $\left[0, \tau_{k}\right]$ with bounded variation for $k=1,2,3$. The limiting covariance matrix of $\sqrt{n}\left(\widehat{\boldsymbol{\theta}}-\boldsymbol{\theta}_{0}\right)$ attains the semiparametric efficiency bound. 
Theorem 4. Under Conditions 1-5 in Section S3 of the online supplement, the estimators for stratum-specific effects given in (2.5)-2.10) are consistent and asymptotically normal.

The proofs of Theorems 3 and 4 are given in Section S3 of the online supplement. Since the form of the limiting variances of the stratum-specific effects is complicated, we estimate the variance of the estimators by a nonparametric bootstrap procedure in all numerical studies.

\section{Simulation Studies}

We conducted simulation studies to examine the performance of the proposed methods. We generated two covariates $X_{1} \sim N(0,1)$ and $X_{2} \sim$ Unif $(0,1)$ and generated the treatment indicator $A \sim \operatorname{Bin}(0.5)$ to reflect 1:1 randomization. We set $\Lambda_{1}(t)=t, \Lambda_{2}(t)=0.2 t$, and $\Lambda_{3}(t)=\log (1+t)$, while the true values of the other parameters are shown in Tables S1 and S2 of the online supplement. We generated a censoring time $C \sim \operatorname{Unif}(0,15)$ to obtain approximately $51 \%$ and $26 \%$ censoring rates for the nonterminal and terminal events, respectively. The proportions of subjects with $U=1,2,3$ are approximately $31 \%, 41 \%$, and $28 \%$, respectively.

We considered 1000 replicates with sample sizes $n=1000$ and 2000, where 100 bootstrap samples were used for variance estimation. All exam- 
ined replications converge with a $10^{-6}$ convergence criterion. The results for the parameter estimators are shown in Tables S1 and S2 and Figure S1 of Section S4.1 of the online supplement. The parameter estimators are virtually unbiased, and the bootstrap variance estimators are accurate as sample size increases.

Table 1 shows the performance of the estimated stratum-specific indirect and direct effects in stratum with $U=1$ and $\boldsymbol{X}=(0.5,0.5)^{\mathrm{T}}$, as well as the estimated total effects for strata with $U=2,3$ and the same covariate values. Similarly, for any $t$ the average was taken over all the replicates with estimators that have last jump time no less than $t$. The bias gets smaller as sample size increases. The variance estimator is accurate and the coverage probability is close to the nominal level when sample size is large.

In Section S4.2 of the online supplement, we evaluate the performance of the proposed approach when the baseline hazard functions are indeed different for different strata. Specifically, we changed the baseline hazard functions for the event times with $U=2$. Even though the total effects in strata with $U=2$ and $U=3$ may be estimated with bias, the bias for the mediation effects in stratum with $U=1$ is relatively small, and the the coverage probabilities for the $95 \%$ confidence intervals are close to the nominal level. 
Table 1: Simulation results for stratum-specific mediation effects and total effects.

\begin{tabular}{|c|c|c|c|c|c|c|c|c|c|c|}
\hline & \multirow[b]{2}{*}{$\mathrm{t}$} & \multirow[b]{2}{*}{ True Value } & \multicolumn{4}{|c|}{$n=1000$} & \multicolumn{4}{|c|}{$n=2000$} \\
\hline & & & Bias & $\mathrm{SE}$ & SEE & $\mathrm{CP}$ & Bias & $\mathrm{SE}$ & SEE & $\mathrm{CP}$ \\
\hline \multirow[t]{3}{*}{$N D E_{1}$} & 2 & -0.11 & 0.014 & 0.056 & 0.070 & 0.97 & 0.007 & 0.031 & 0.041 & 0.97 \\
\hline & 4 & -0.17 & 0.018 & 0.090 & 0.106 & 0.96 & 0.010 & 0.052 & 0.066 & 0.97 \\
\hline & 6 & -0.18 & 0.020 & 0.096 & 0.107 & 0.94 & 0.010 & 0.057 & 0.069 & 0.96 \\
\hline \multirow[t]{3}{*}{$N I E_{1}$} & 2 & -0.04 & -0.001 & 0.022 & 0.025 & 0.97 & 0.000 & 0.015 & 0.017 & 0.97 \\
\hline & 4 & -0.03 & -0.001 & 0.016 & 0.018 & 0.97 & -0.001 & 0.011 & 0.012 & 0.96 \\
\hline & 6 & -0.02 & -0.001 & 0.010 & 0.011 & 0.97 & 0.000 & 0.007 & 0.007 & 0.96 \\
\hline \multirow[t]{4}{*}{$T E_{2}$} & 2 & -0.10 & -0.036 & 0.158 & 0.175 & 0.97 & -0.022 & 0.115 & 0.126 & 0.97 \\
\hline & 4 & 0.10 & -0.046 & 0.159 & 0.180 & 0.96 & -0.026 & 0.113 & 0.127 & 0.97 \\
\hline & 6 & 0.17 & -0.047 & 0.136 & 0.156 & 0.97 & -0.027 & 0.097 & 0.109 & 0.97 \\
\hline & 8 & 0.18 & -0.044 & 0.115 & 0.131 & 0.96 & -0.025 & 0.084 & 0.093 & 0.96 \\
\hline \multirow[t]{4}{*}{$T E_{3}$} & 2 & -0.07 & 0.021 & 0.139 & 0.147 & 0.97 & 0.015 & 0.117 & 0.114 & 0.95 \\
\hline & 4 & -0.06 & 0.031 & 0.119 & 0.126 & 0.98 & 0.022 & 0.101 & 0.099 & 0.96 \\
\hline & 6 & -0.06 & 0.033 & 0.101 & 0.107 & 0.97 & 0.024 & 0.086 & 0.085 & 0.96 \\
\hline & 8 & -0.05 & 0.033 & 0.088 & 0.093 & 0.97 & 0.024 & 0.075 & 0.074 & 0.96 \\
\hline
\end{tabular}

\section{Application}

We consider application of the proposed methods to a prostate cancer clinical trial. NCIC Clinical Trials Group PR.3/Medical Research Coun- 
cil PR07/Intergroup T94-0110 is a randomized controlled trial of patients with locally advanced prostate cancer. The primary objective is to determine whether the addition of radiotherapy (RT) to androgen-deprivation therapy (ADT) prolonged overall survival, defined as time from random assignment to death from any cause. One thousand two hundred and five patients with locally advanced prostate cancer were recruited and randomly assigned between 1995 and 2005, 602 to ADT alone and 603 to ADT + RT. In the final report of the study (Mason et al., 2015), at a median follow-up time of 8 years, 465 patients had died. Overall survival was significantly improved in the patients allocated to ADT + RT (hazard ratio 0.70 with 95\% CI, 0.57 to $0.85 ; \mathrm{P}<.001)$.

In addition to the primary outcome of death, the study also collected data on time to disease progression, which was defined as the first of any of the following events: biochemical progression, local progression, or development of metastatic disease. We analyzed the data to reveal the proportions of the treatment effect on overall survival that are mediated by disease progression. Particularly, we adjusted for initial PSA level ( $<20$ vs. 20 to 50, vs. $>50 \mathrm{~g} / \mathrm{L})$ and Gleason score( 8 vs. 8 to 10$)$.

We analyzed the data using the proposed approach, with 1000 bootstrap samples for variance estimation. The parameter estimates for regres- 
sion coefficients for the event time processes are shown in Table 2, For stratum with $U=1$, ADT + RT is associated with a decreased risk of disease progression, while it is associated with an increased risk from disease progression to death. For stratum with $U=3$, ADT + RT is associated with a decreased risk of death. The effects are not significant at 0.05 level. For stratum with $U=1$, a subject with initial PSA level $>50 \mathrm{~g} / \mathrm{L}$ is associated with significantly increased risk of disease progression, compared to a similar subject with initial PSA level $<20 \mathrm{~g} / \mathrm{L}$; and a subject with Gleason score 8-10 is associated with significantly decreased risk of disease progression, compared to a similar subject with Gleason score $<8$.

Table 3 shows the parameter estimators of the logistic regression model for stratum membership. By averaging over the stratum membership probabilities over all subjects given their covariate values, the average probabilities of belong to strata $U=1,2$, and 3 are $40.1 \%, 25.7 \%$, and $34.2 \%$, respectively. To verify if the model is reasonable, we estimated the stratumspecific survival functions for every subject and summarize the subjectspecific survival function by weighting them by his/her stratum membership probabilities. We average the estimated survival functions for subjects assigned to ADT+RT versus ADT, and plot them against the survival function estimators from the Kaplan Meier methods and the proportional haz- 
Table 2: Parameter estimates for regression coefficients of event times.

\begin{tabular}{|c|c|c|c|c|c|c|}
\hline \multirow{3}{*}{ Process } & \multicolumn{6}{|c|}{$U=1$} \\
\hline & \multicolumn{3}{|c|}{ Health $\rightarrow$ Disease } & \multicolumn{3}{|c|}{ Disease $\rightarrow$ Death } \\
\hline & Est & SEE & $p$-value & Est & SEE & $p$-value \\
\hline \multirow{3}{*}{$\begin{array}{l}\text { Initial PSA Level }(20 \text { to } 50 \mathrm{~g} / \mathrm{L}) \\
\text { Initial PSA Level }(>50 \mathrm{~g} / \mathrm{L})\end{array}$} & -0.825 & 0.925 & 0.373 & 0.460 & 0.569 & 0.419 \\
\hline & 0.321 & 0.563 & 0.569 & -0.097 & 0.322 & 0.763 \\
\hline & 1.607 & 0.614 & 0.009 & 0.065 & 0.337 & 0.846 \\
\hline Gleason Score (8-10) & -2.008 & 0.478 & $<0.0001$ & -0.378 & 0.274 & 0.167 \\
\hline \multirow{3}{*}{ Process } & \multicolumn{6}{|c|}{$U=2, \mathrm{ADT}$} \\
\hline & \multicolumn{3}{|c|}{ Health $\rightarrow$ Disease } & \multicolumn{3}{|c|}{ Disease $\rightarrow$ Death } \\
\hline & Est & SEE & $p$-value & Est & SEE & $p$-value \\
\hline \multirow{4}{*}{$\begin{array}{r}\text { Initial PSA Level }(20 \text { to } 50 \mathrm{~g} / \mathrm{L}) \\
\text { Initial PSA Level }(>50 \mathrm{~g} / \mathrm{L}) \\
\text { Gleason Score }(8-10)\end{array}$} & -1.917 & 1.534 & 0.211 & -0.557 & 2.490 & 0.823 \\
\hline & 0.663 & 0.985 & 0.501 & -0.304 & 2.007 & 0.880 \\
\hline & 1.619 & 0.967 & 0.094 & -0.104 & 1.693 & 0.951 \\
\hline & 0.674 & 0.932 & 0.469 & -0.106 & 1.638 & 0.948 \\
\hline \multirow{3}{*}{ Process } & \multicolumn{3}{|c|}{$U=2, \mathrm{ADT}+\mathrm{DT}$} & \multicolumn{3}{|c|}{$U=3$} \\
\hline & \multicolumn{3}{|c|}{ Health $\rightarrow$ Death } & \multicolumn{3}{|c|}{ Health $\rightarrow$ Death } \\
\hline & Est & SEE & $p$-value & Est & SEE & $p$-value \\
\hline Intercept & -3.212 & 7.225 & 0.657 & -0.446 & 0.827 & 0.590 \\
\hline Initial PSA Level (20 to $50 \mathrm{~g} / \mathrm{L}$ ) & 1.146 & 4.886 & 0.815 & -0.022 & 0.602 & 0.970 \\
\hline Initial PSA Level (> $50 \mathrm{~g} / \mathrm{L})$ & 1.601 & 4.937 & 0.746 & -0.883 & 0.641 & 0.168 \\
\hline Gleason Score (8-10) & 1.624 & 5.266 & 0.758 & -0.514 & 0.484 & 0.289 \\
\hline
\end{tabular}


Table 3: Parameter estimates for regression coefficients of stratum membership

\begin{tabular}{|rccccccc|}
\hline & \multicolumn{3}{c}{$\boldsymbol{\alpha}_{1}$} & \multicolumn{4}{c|}{$\boldsymbol{\alpha}_{2}$} \\
\hline & Est & SEE & $p$-value & Est & SEE & $p$-value \\
\hline Intercept & 0.205 & 0.433 & 0.636 & 0.291 & 0.611 & 0.634 \\
Initial PSA Level (20 to 50 g/L) & 0.090 & 0.562 & 0.872 & 0.625 & 0.898 & 0.486 \\
Initial PSA Level (> 50 g/L) & -0.684 & 0.541 & 0.206 & 0.004 & 0.816 & 0.996 \\
Gleason Score (8-10) & 0.134 & 0.408 & 0.742 & -1.495 & 0.771 & 0.053 \\
\hline
\end{tabular}

ards model. The results are shown in Figure 1. The estimated populationaverage survival functions for ADT $+\mathrm{RT}$ and ADT groups are similar to those from the Kaplan Meier methods and the proportional hazards model, especially for time before 10 years when data are not sparse, indicating proper fit of the proposed approach.

Figure 2 shows the estimated marginalized stratum-specific indirect and direct effects (with $95 \%$ confidence intervals) for stratum with $U=1$. The estimated natural indirect effect is positive and increasing over time, and the estimated natural direct effect is slightly negative over time. However, the $95 \%$ confidence intervals are wide such that the stratum-specific natural indirect and direct effects are not significant different from zero. The total effect in stratum with $U=1$ is positive and increasing over time, corre- 


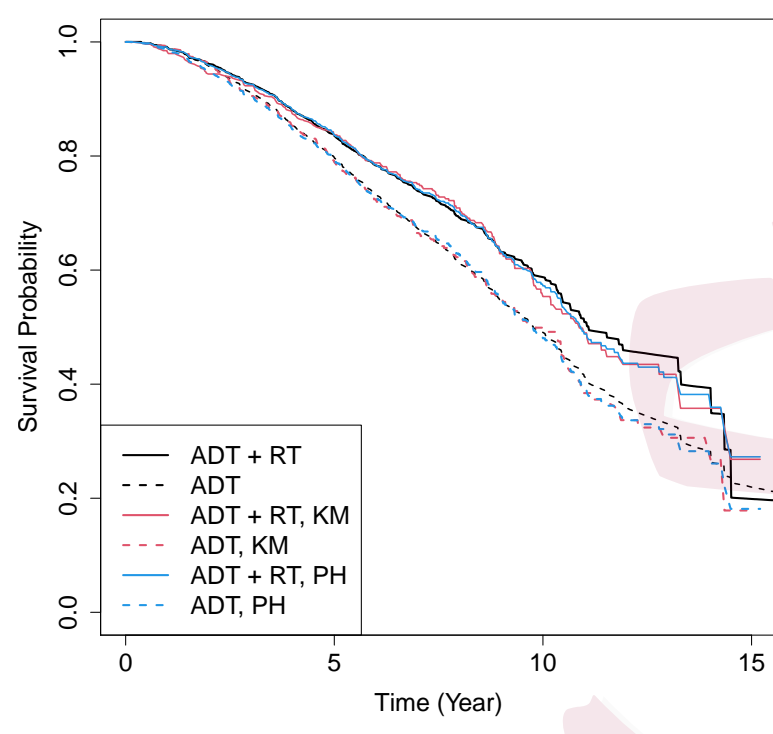

Figure 1: Estimated survival functions from the proposed, Kaplan-Meier, and proportional hazards model approaches.

sponding to an increased survival probability assigned to ADT + RT versus ADT in stratum with $U=1$.

\section{Discussion}

Semi-competing risks data are frequently observed in medical studies, where the terminal event time may censor the intermediate event time but not vice versa. To define and estimate causal contrasts of the effect of a treatment to the terminal and intermediate events, we introduced a novel principal 


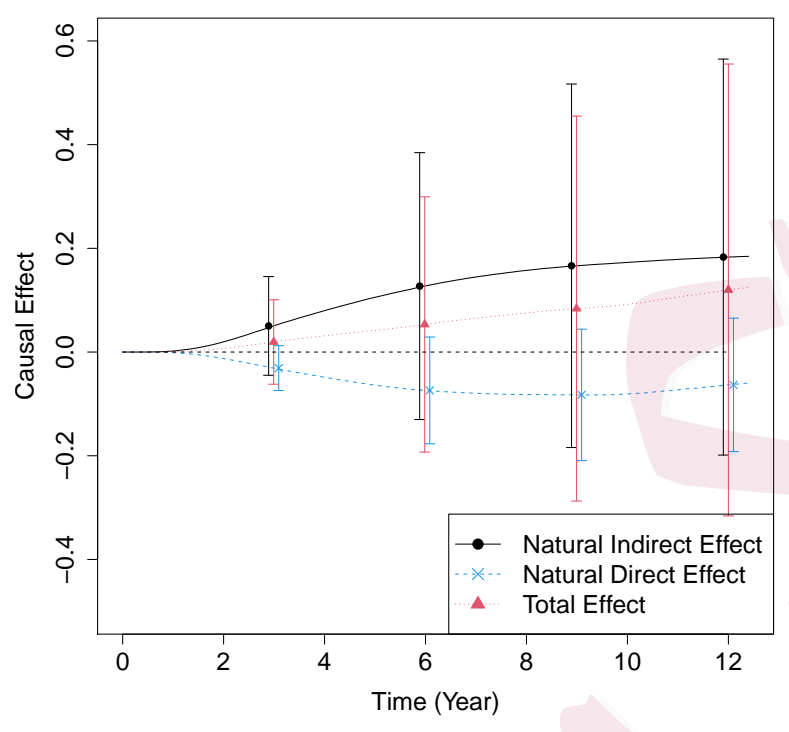

Figure 2: Estimated stratum-specific indirect and direct effects in stratum with $U=1$.

stratification framework that distinguishes susceptible and non-susceptible subjects given different treatments, and defined the natural indirect and direct effects in the stratum where the times to intermediate and terminal events are well-defined given both treatments. We provided reasonable assumptions to identify the stratum-specific natural indirect and direct effects, proposed a semiparametric model, and studied an EM algorithm to obtain the nonparametric maximum likelihood estimators of model parameters. We showed that the estimators are consistent and asymptotically efficient estimated under mild regularity conditions, and their performance 
are satisfactory in finite sample numerical studies.

In identifying the stratum-specific natural indirect and direct effects, we assumed that there are no subjects who are susceptible to the intermediate event under treatment $(A=1)$ and non-susceptible under control $(A=0)$. This assumption may need careful examination based on scientific understanding of how treatment may affect the intermediate event. In our data application, we assessed this assumption by fitting the proposed model with switched treatment indicator labels of ADT+RT and ADT. The estimated probability of belonging to stratum with $U=2$ (equivalent to the fourth stratum in the original labeling) is very low (6.9\%), suggesting that the assumption on non-existence of the fourth stratum may be valid. In Section S4.3 of the online supplement, we have included a sensitivity analysis assessing the performance of the estimator for stratum-specific effects when there is a fourth stratum with small probability $(\sim 6.9 \%)$. Even though the assumption on non-existence of the fourth stratum fails, the stratum-specific effects in the first three strata can still be estimated with relatively small bias. In some applications, this fourth stratum may indeed exist. In the literature of principal stratification for uncensored data with four or more strata, the effect of interest often can only be interval identified. Interval identification with a regression model often results in a 
complicated solution manifold, with properties often not well understood. We plan to explore this problem in a future study. The proposed nonparametric maximum likelihood estimation framework relies on the validity of the modeling assumptions, including the proportional hazards assumption on the hazard functions. Even though model checking for mixture model for right-censored data has been considered in literature (e.g., Peng and Taylor (2017)), model checking of semi-competing risks data may not available for illness-death model. A model checking procedure on the modeling assumptions may not be trivial and would require further research.

\section{Supplementary Materials}

Supplementary Materials include proofs of Theorems 1 and 2 referenced in Section 2.3, details on EM algorithm referenced in Section 2.5, other proofs of asymptotic results referenced in Section 2.6, and additional simulation results referenced in Sections 3 and 5 .

\section{Acknowledgements}

This manuscript was prepared using data from Dataset NCT00002633-D1 from the NCTN Data Archive of the National Cancer Institute (NCI) National Clinical Trials Network (NCTN). Data were originally collected from 
REFERENCES

clinical trial NCT00002633 Phase III Randomized Trial Comparing Total Androgen Blockade Versus Total Androgen Blockade Plus Pelvic Irradiation in Clinical Stage T3-4, N0, M0 Adenocarcinoma of the Prostate All analyses and conclusions in this manuscript are the sole responsibility of the authors and do not necessarily reflect the opinions or views of the clinical trial investigators, the NCTN, or the NCI. The authors are partially funded by the U.S. National Institutes of Health grants R01HL122212, U01AG016976 and U.S. National Science Foundation grant DMS 1711952.

\section{References}

Aalen, O. O., M. J. Stensrud, V. Didelez, R. Daniel, K. Røysland, and S. Strohmaier (2020). Time-dependent mediators in survival analysis: Modeling direct and indirect effects with the additive hazards model. Biometr. J. 62(3), 532-549.

Angrist, J. D., G. W. Imbens, and D. B. Rubin (1996). Identification of causal effects using instrumental variables. J. Am. Stat. Assoc. $91(434), 444-455$.

Comment, L., F. Mealli, S. Haneuse, and C. Zigler (2019). Survivor average causal effects for continuous time: a principal stratification approach to causal inference with semicompeting risks. arXiv preprint arXiv:1902.09304.

Copelan, E. A., J. C. Biggs, J. M. Thompson, P. Crilley, J. Szer, J. P. Klein, N. Kapoor, B. R. Avalos, I. Cunningham, and K. Atkinson (1991). Treatment for acute myelocytic 


\section{REFERENCES}

leukemia with allogeneic bone marrow transplantation following preparation with BuCy2. Blood 78(3), 838-843.

Didelez, V. (2019). Defining causal mediation with a longitudinal mediator and a survival outcome. Lifetime Data Anal. 25(4), 593-610.

Fine, J. P., H. Jiang, and R. Chappell (2001). On semi-competing risks data. Biometrika 88(4), 907-919.

Frangakis, C. E. and D. B. Rubin (2002). Principal stratification in causal inference. Biometrics $58(1), 21-29$.

Huang, Y.-T. (2020). Causal mediation of semicompeting risks. Biometrics.

Imai, K., L. Keele, and T. Yamamoto (2010). Identification, inference and sensitivity analysis for causal mediation effects. Stat. Sci. 25(1), 51-71.

Klein, J. P. and M. L. Moeschberger (2006). Survival Analysis: Techniques for Censored and Truncated Data. New York: Springer.

Lange, T. and J. V. Hansen (2011). Direct and indirect effects in a survival context. Epidemiol. 22(4), 575-581.

Lange, T., S. Vansteelandt, and M. Bekaert (2012). A simple unified approach for estimating natural direct and indirect effects. Am. J. Epidemiol. 176(3), 190-195.

Lin, D., W. Sun, and Z. Ying (1999). Nonparametric estimation of the gap time distribution for serial events with censored data. Biometrika $86(1), 59-70$. 


\section{REFERENCES}

Lin, S.-H., J. G. Young, R. Logan, and T. J. VanderWeele (2017). Mediation analysis for a survival outcome with time-varying exposures, mediators, and confounders. Stat. Med. 36(26), 4153-4166.

Maller, R. A. and S. Zhou (1992). Estimating the proportion of immunes in a censored sample. Biometrika $79(4), 731-739$.

Mason, M. D., W. R. Parulekar, M. R. Sydes, M. Brundage, P. Kirkbride, M. Gospodarowicz, R. Cowan, E. C. Kostashuk, J. Anderson, G. Swanson, et al. (2015). Final report of the intergroup randomized study of combined androgen-deprivation therapy plus radiotherapy versus androgen-deprivation therapy alone in locally advanced prostate cancer. J. Clin. Oncol. 33(19), 2143-2150.

Nevo, D. and M. Gorfine (2020). Causal inference for semi-competing risks data. arXiv preprint arXiv:2010.04485.

Peng, Y. and J. M. Taylor (2017). Residual-based model diagnosis methods for mixture cure models. Biometrics 73(2), 495-505.

Tchetgen Tchetgen, E. J. (2011). On causal mediation analysis with a survival outcome. Int. J. Biostat. 7(1), 1-38.

VanderWeele, T. J. (2011). Causal mediation analysis with survival data. Epidemiol. 22(4), 582-585.

Vansteelandt, S., M. Linder, S. Vandenberghe, J. Steen, and J. Madsen (2019). Mediation analysis of time-to-event endpoints accounting for repeatedly measured mediators subject 
to time-varying confounding. Stat. Med. 38(24), 4828-4840.

Wang, W. and M. T. Wells (1998). Nonparametric estimation of successive duration times under dependent censoring. Biometrika 85(3), 561-572.

Xu, J., J. D. Kalbfleisch, and B. Tai (2010). Statistical analysis of illness-death processes and semicompeting risks data. Biometrics $66(3), 716-725$.

Xu, Y., D. Scharfstein, P. Müller, and M. Daniels (2020). A bayesian nonparametric approach for evaluating the causal effect of treatment in randomized trials with semi-competing risks. Biostatistics, kxaa008.

Yu, W., K. Chen, M. E. Sobel, and Z. Ying (2015). Semiparametric transformation models for causal inference in time-to-event studies with all-or-nothing compliance. J. R. Stat. Soc. $B 77(2), 397-415$.

Zeng, D. and D. Lin (2007). Maximum likelihood estimation in semiparametric regression models with censored data. J. R. Stat. Soc. B 69(4), 507-564.

Zhang, J. L. and D. B. Rubin (2003). Estimation of causal effects via principal stratification when some outcomes are truncated by "death". J. Educ. Behav. Stat. 28(4), 353-368.

Zheng, W. and M. van der Laan (2017). Longitudinal mediation analysis with time-varying mediators and exposures, with application to survival outcomes. J. Causal Inference 5(2), $1-24$.

Vaccine and Infectious Diseases Division, Fred Hutchinson Cancer Research Center, Seattle, 
Washington, 98109, U.S.A.

E-mail: fgao@fredhutch.org

National Alzheimer's Coordinating Center, University of Washington, Seattle, Washington,

98195, U.S.A.

E-mail: fanxia@uw.edu

Department of Biostatistics, University of Washington, Seattle, Washington, 98195, U.S.A.

E-mail: kcgchan@u.washington.edu 